\title{
Erratum to: Environmental impacts of German food consumption and food losses
}

\author{
Ulrike Eberle $^{1} \cdot$ Jacob Fels $^{2}$
}

Published online: 10 March 2016

(C) Springer-Verlag Berlin Heidelberg 2016

Erratum to: International Journal of Life Cylce Assessment

DOI 10.1007/s11367-015-0983-7

This is a corrigendum and clarification on behalf of the authors. Table 3 in the original version of this article has been corrected as follows:

The online version of the original article can be found at http://dx.doi.org/ 10.1007/s11367-015-0983-7.

Ulrike Eberle

u.eberle@corsus.de

1 corsus-corporate sustainability, Nernstweg 32-34, 22765 Hamburg, Germany

2 Zentrum für Nachhaltige Unternehmensführung, Universität Witten-Herdecke, Alfred-Herrhausen-Str. 50,

58448 Witten, Germany 
Table 3 Amounts of purchased food, eaten food and food losses per food item analyzed for out of home consumption

\begin{tabular}{|c|c|c|c|c|}
\hline Product group & $\begin{array}{l}\text { Food purchases } \\
{[\mathrm{kg} / \mathrm{cap}]}\end{array}$ & $\begin{array}{l}\text { Consumption } \\
{[\mathrm{kg} / \mathrm{cap}]}\end{array}$ & Losses [kg/cap] & Assumptions \\
\hline Bread and cereals & 28.295 & 18.818 & 9.478 & \\
\hline Rice & 0.721 & 0.479 & 0.241 & \\
\hline Bread and backed goods & 20.650 & 13.733 & 6.917 & \\
\hline Pasta and other cereals & 6.925 & 4.605 & 2.319 & \\
\hline Meat and meat products & 9.820 & 6.531 & 3.289 & \\
\hline Bovine and veal meat & 2.707 & 1.801 & 0.907 & Incl. sheep, goats, other meat, analyzed as beef \\
\hline Pork meat & 2.702 & 1.797 & 0.905 & Incl. meat without specification, analyzed as pork \\
\hline Poultry meat & 2.046 & 1.361 & 0.685 & analyzed as broiler \\
\hline Meat products & 2.364 & 1.572 & 0.792 & $\begin{array}{l}\text { Incl. Meat preparations, analyzed as pork, broiler } \\
\text { and beef meat with a further processing step }\end{array}$ \\
\hline Fish and fish products & 2.076 & 1.381 & 0.695 & analyzed as wild fish \\
\hline Dairy products and eggs & 7.902 & 5.255 & 2.647 & \\
\hline Milk & 5.650 & 3.757 & 1.892 & $\begin{array}{l}\text { Incl. milks and preserved milks produced and other } \\
\text { milk and cream based products, incl. yoghurt }\end{array}$ \\
\hline Cheese & 0.770 & 0.512 & 0.258 & Incl. cheese without specification \\
\hline Cream & 0.220 & 0.146 & 0.074 & \\
\hline Butter & 0.196 & 0.130 & 0.066 & \\
\hline Eggs & 1.067 & 0.710 & 0.357 & \\
\hline Fats an oils & 6.972 & 4.637 & 2.335 & w/o butter \\
\hline Fruits & 2.441 & 1.624 & 0.818 & \\
\hline Citrus fruits & 0.406 & 0.270 & 0.136 & analyzed as oranges \\
\hline Bananas & 0.593 & 0.395 & 0.199 & Incl. other tropical fruits \\
\hline Apples & 1.351 & 0.898 & 0.452 & $\begin{array}{l}\text { Incl. pears. drupes, pome, berries, grapes, nuts, seeds, } \\
\text { dried fruits and fruits without specification }\end{array}$ \\
\hline Canned and frozen fruits & 0.091 & 0.061 & 0.031 & analyzed as canned and frozen apples \\
\hline Vegetables and potatoes & 12.776 & 8.497 & 4.279 & \\
\hline Tomatoes & 2.012 & 1.338 & 0.674 & Incl. capsicum, cucumber and other fruit vegetables \\
\hline Fresh vegetables and salad & 3.060 & 2.035 & 1.025 & \\
\hline Dry, frozen and canned vegetables & 4.460 & 2.966 & 1.494 & analyzed as fresh vegetables \\
\hline Potatoes & 3.244 & 2.158 & 1.087 & \\
\hline Sugar & 0.188 & 0.125 & 0.063 & Incl. sweetener \\
\hline SUM & 70.471 & 46.866 & 23.605 & \\
\hline
\end{tabular}

